Physics

Physics Research Publications

Purdue University

Year 2006

\title{
Spontaneous spin polarization in quantum point contacts
}

\author{
L. P. Rokhinson \\ L. N. Pfeiffer
}

K. W. West

This paper is posted at Purdue e-Pubs.

http://docs.lib.purdue.edu/physics_articles/221 


\title{
Spontaneous Spin Polarization in Quantum Point Contacts
}

\author{
L. P. Rokhinson, ${ }^{1}$ L. N. Pfeiffer, ${ }^{2}$ and K.W. West ${ }^{2}$ \\ ${ }^{1}$ Department of Physics, Purdue University, West Lafayette, Indiana 47907 USA \\ ${ }^{2}$ Bell Laboratories, Lucent Technologies, Murray Hill, New Jersey 07974 USA
}

(Received 27 January 2006; published 21 April 2006)

\begin{abstract}
We use spatial spin separation by a magnetic focusing technique to probe the polarization of quantum point contacts. The point contacts are fabricated from $p$-type GaAs/AlGaAs heterostructures. A finite polarization is measured in the low-density regime, when the conductance of a point contact is tuned to $<2 e^{2} / h$. Polarization is stronger in samples with a well-defined " 0.7 structure."
\end{abstract}

DOI: 10.1103/PhysRevLett.96.156602

Mesoscopic systems exhibit a range of nontrivial spinrelated phenomena in the low-density regime, where interparticle Coulomb interactions become comparable to their kinetic energy. In zero-dimensional systems the spontaneous polarization of a few-electron quantum dot leads to spin blockade [1-3], a remarkable effect where the mismatch of a single spin blocks macroscopic current flow. In two-dimensional hole gases there is experimental evidence of a finite spin polarization even in the absence of a magnetic field [4]. In one-dimensional systems - quantum wires and quantum point contacts - a puzzling so-called "0.7 structure" has been observed below the first quantization plateau [5]. Experiments suggest [5-8] that an extra plateau in the conductance vs gate voltage characteristic at $0.7 \times 2 e^{2} / h$ is spin related; however, the origin of the phenomenon is not yet understood and is highly debated. In this Letter we report direct measurements of finite polarization of holes in a quantum point contact (QPC) at conductances $G<2 e^{2} / h$. We incorporated a QPC into a magnetic focusing device [9] so that the polarization can be measured directly using a recently developed spatial spin separation technique [10].

Quantization of the ballistic conductance $G$ in integer multiples of $g_{0}=2 e^{2} / h$ is a fundamental property of $1 \mathrm{D}$ systems $[11,12]$, which originates from the exact cancellation of velocity and the 1D density of states. Each energy level below the Fermi energy inside a 1D channel contributes $0.5 g_{0}$ to the total conductance, and an extra factor of 2 accounts for the spin degeneracy. Strong magnetic fields can lift the degeneracy; in this case quantization in multiples of $0.5 g_{0}$ is observed. This single-particle result is robust even in the presence of electron-electron interactions because they preserve the center-of-mass velocity of the scattered electrons. Thus, the observation of a quantized plateau at $0.7 g_{0}$ in the absence of magnetic field in $n$-GaAs [5], $p$-Si [13], $n$-GaN [14], and $p$-GaAs QPCs, as well as in long clean 1D wires [15], poses a serious challenge to our understanding of 1D conductors.

Phenomenologically, the observed structure can be explained if one assumes the existence of a static spin polarization at zero magnetic field and confinement-dependent spin splitting of the spin subbands [16]. However, the well-
PACS numbers: 72.25.-b, 71.70.Ej, 73.23.Ad, 85.75.-d

known Lieb-Mattis theorem forbids polarization in 1D systems [17]. Some theories suggest a possible deviation from this theorem in a realistic channel with finite width [18-20]. Recently, it has been pointed out that the temperature and bias dependence of the differential conductance around the $0.7 g_{0}$ plateau are similar to the Kondo phenomenon, thus suggesting dynamic spin polarization [7,21]. Alternative theories assume no polarization and attribute the phenomenon to electron-phonon interactions [22] or to the formation of a Wigner crystal [23]. None of the above theories describe the variety of observed phenomena in a unified and consistent fashion. Thus, direct measurement of the spin polarization becomes of paramount importance.

Our devices are fabricated from a two-dimensional hole gas (2DHG) using an atomic force microscopy (AFM) local anodic oxidation technique [24,25]. Oxide lines separate the 2DHG underneath by forming $\sim 200 \mathrm{mV}$ potential barriers. Several specially designed heterostructures are grown by molecular-beam epitaxy technique on [113]A GaAs [26]. Despite its very close proximity to the surface $(350 \AA)$, the 2DHG has exceptionally high mobility $\sim 0.5 \times 10^{6} \mathrm{~V} \mathrm{~s} / \mathrm{cm}^{2}$. The devices are fabricated from two wafers with hole densities $p=1.47 \times 10^{11} \mathrm{~cm}^{-2}$ (wafer A) and $p=0.9 \times 10^{11} \mathrm{~cm}^{-2}$ (wafer B). For quantitative analysis we use data collected during a single cooldown for each device. The qualitative features are reproducible upon several thermal cyclings.

The devices consist of two QPCs separated by a central gate (see inset in Fig. 1). The potential inside the point contacts can be controlled separately by the two side gates $V_{g 1}$ and $V_{g 2}$, or by the central gate $V_{\mathrm{gc}}$. The conductance of point contact QPC1 is plotted in Fig. 1 as a function of the gate voltage $V_{g 1}$. At zero field (left-most curve), plateaus with conductance quantized at $g_{0}$ and $2 g_{0}$ are clearly observed. In addition, an extra plateau can be seen at $G \sim$ $0.7 g_{0}$ and, less developed, at $G \sim 1.7 g_{0}$. When an in-plane magnetic field $B_{\|}$is applied, the $0.7 g_{0}$ and $1.7 g_{0}$ plateaus gradually shift toward $0.5 g_{0}$ and $1.5 g_{0}$, saturating for $B_{\|}>$ $4 \mathrm{~T}$. This gradual decrease is different from the abrupt appearance of half-integer plateaus for higher energy levels. In that case the plateaus become more prominent as the 


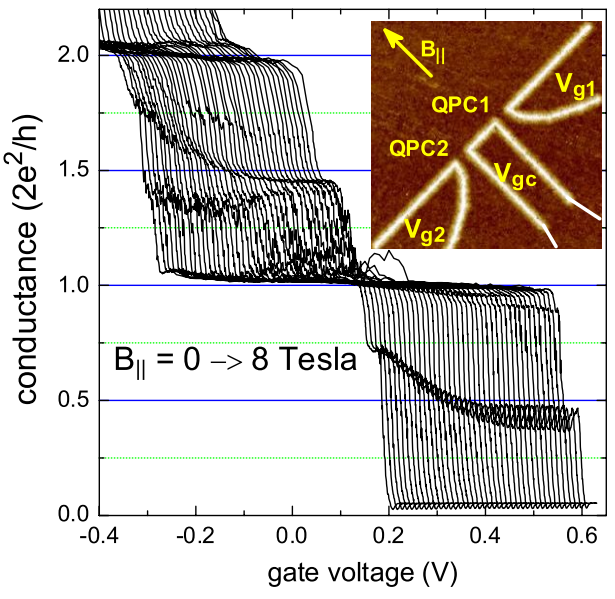

FIG. 1 (color online). The conductance of a quantum point contact $G$ is plotted as a function the gate voltage $V_{g 1}$ for inplane magnetic fields $0<B_{\|}<8 \mathrm{~T}$ at temperature $T=50 \mathrm{mK}$ for QPC1. The curves are offset proportionally to $B_{\|}$, the left most is $B_{\|}=0$. Inset: AFM micrograph of a sample $(3.3 \mu \mathrm{m} \times$ $3.3 \mu \mathrm{m})$. Light lines are the oxide which separates different regions of the $2 \mathrm{D}$ hole gas. The two point contacts QPC1 and QPC2 form a magnetic focusing device. The conductance of the QPCs is controlled via voltages applied to the gates $V_{g 1}, V_{g 2}$, and the central gate $V_{\mathrm{gc}}$. The direction of $B_{\|}$is indicated by an arrow.

Zeeman splitting increases, but the conductance values of the plateaus do not change with $B_{\|}$, consistent with the single-particle picture.

Another signature of the 0.7 structure is the anomalous nonlinear differential conductance $g=d I / d V$. A distinct peak in $g$ vs dc bias $V_{\text {bias }}$ has been reported in electron QPCs [7]. The nonlinear conductance in our hole device is analyzed in Fig. 2. Indeed, there is a well developed zerobias peak at the lowest $T=25 \mathrm{mK}$ and $B_{\|}=0$. The peak is suppressed if $T$ or $B_{\|}$are increased. $g(T)$ and $g\left(B_{\|}\right)$at $V_{\text {bias }}=0$ are plotted in Figs. 2(c) and 2(d). A zero-bias peak and its suppression by $T$ and $B_{\|}$is a hallmark of the Kondo phenomenon. The Lande factor $g^{*} \approx 0.3$ in the point contact is too small to result in a detectable Zeeman splitting of the zero-bias anomaly in our samples.

Experimentally, it is possible to clarify the origin of the 0.7 structure by measuring the polarization of carriers emerging from the QPC. The polarization can be measured in a ballistic magnetic focusing device with spin-orbit interaction, where carriers with opposite spin have different cyclotron orbits in small external magnetic field [10]. In the presence of spin-orbit interaction, carriers with the same energy and opposite spin orientation have different momenta $\hbar k^{ \pm}=\sqrt{2 m\left(e_{f}-\gamma^{2} / m\right)} \pm \gamma$, where $e_{f}=$ $2 \pi p \hbar^{2} / m$ is the Fermi energy, $\hbar$ is Plank's constant, $p$ is 2D hole density, $m$ is the effective mass, and $\gamma$ is a spinorbit interaction constant, $\gamma / \hbar \approx 10^{-3} \AA$ in our samples. Thus, in a weak orbital magnetic field $B_{\perp}$, carriers with opposite spins injected from a QPC will have different
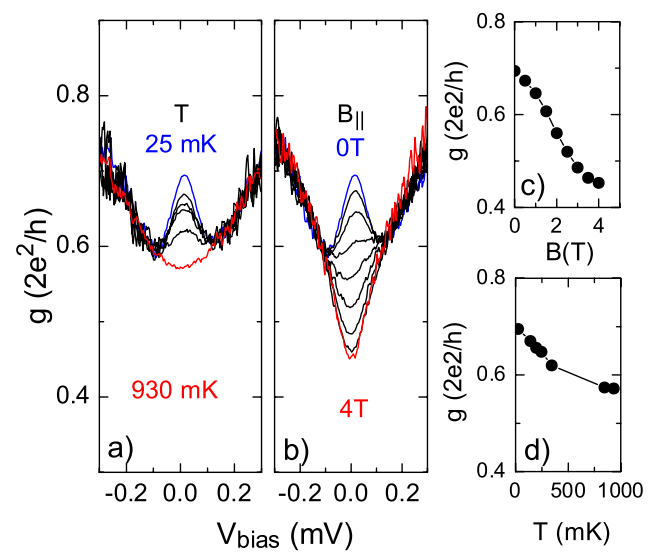

FIG. 2 (color online). Differential conductance $g=d I / d V$ is measured as a function of dc bias $V_{\text {bias }}$ across the QPC1. Gate voltage $V_{g 1}$ is fixed in the middle of the $0.7 \times 2 e^{2} / h$ plateau at $T=25 \mathrm{mK}$ and $B_{\|}=V_{\text {bias }}=0$. In (a) $B_{\|}=0$ and $T=25,140$, 190, 250, 340, and $930 \mathrm{mK}$, in (b) $T=25 \mathrm{mK}$ and $B_{\|}$changes between 0 and $4 \mathrm{~T}$ in steps of $0.5 \mathrm{~T}$. Zero-bias anomaly is the strongest at the lowest $T$ and $B_{\|}=0$ and is suppressed as $T$ and/ or $B_{\|}$increases. (c), (d) $B$ and $T$ dependence of $g$ at $V_{\text {bias }}=0$.

cyclotron radii $r_{c}^{ \pm}=\hbar k^{ \pm} / e B_{\perp}$ and can be selectively focused into the detector QPC at $B_{\perp}^{ \pm}=\hbar k^{ \pm} / 2 e L$ and measured separately ( $L$ is the distance between the injector and the detector QPCs).

Magneto-focusing data are plotted in Fig. 3(a). Current is injected through QPC1 and the voltage is monitored across the detector QPC2. At $\left|B_{\perp}\right|>0.25 \mathrm{~T}$, Shubnikovde Haas oscillations in the adjacent 2D hole gas are observed. At $B_{\perp}>0$ extra peaks due to magnetic focusing are superimposed on Shubnikov-de Haas oscillations and the first two peaks are clearly observed. The positions of the peaks are close to the expected values for a QPC1QPC2 separation of $L=0.8 \mu \mathrm{m}$ and scale with the 2D hole density as $\sqrt{p_{A} / p_{B}}=1.2$ for the devices fabricated from wafers $\mathrm{A}$ and $\mathrm{B}$. The two peaks within the first focusing peak correspond to the focusing conditions for the two orthogonal spin states in the 2DHG and are adiabatically related to the pure spin states inside the point contacts [10].

The height of the focusing peaks $V_{d}=\alpha G_{d}^{-1} I_{i}(1+$ $\left.P_{i} P_{d}\right)$ is proportional to the total injector current $I_{i}=I^{\uparrow}+$ $I^{\downarrow}$, the polarization of the injector $P_{i}=\left(I^{\dagger}-I^{\downarrow}\right) /\left(I^{\dagger}+I^{\downarrow}\right)$ and the detector $P_{d}=\left(T^{\uparrow}-T^{\downarrow}\right) /\left(T^{\uparrow}+T^{\downarrow}\right)$, and the efficiency of the current transfer $\alpha$ between the injector and the detector [27]. Our case of spatial spin separation corresponds to $P_{d}^{\dagger}=+1$ and $P_{d}^{\downarrow}=-1$ for the two peaks. Thus, the injector polarization can be extracted as $P_{i}=$ $\left(V_{d}^{\uparrow}-V_{d}^{\downarrow}\right) /\left(V_{d}^{\uparrow}+V_{d}^{\downarrow}\right)$, where $V_{d}^{\uparrow}$ and $V_{d}^{\downarrow}$ are measured at the same $G_{i}$, or, alternatively, as $P_{i}=\left|V_{d}^{\sigma} / V_{d 0}^{\sigma}-1\right|$ for each peak, where $V_{d 0}^{\sigma}$ is the corresponding peak height for unpolarized injection. 


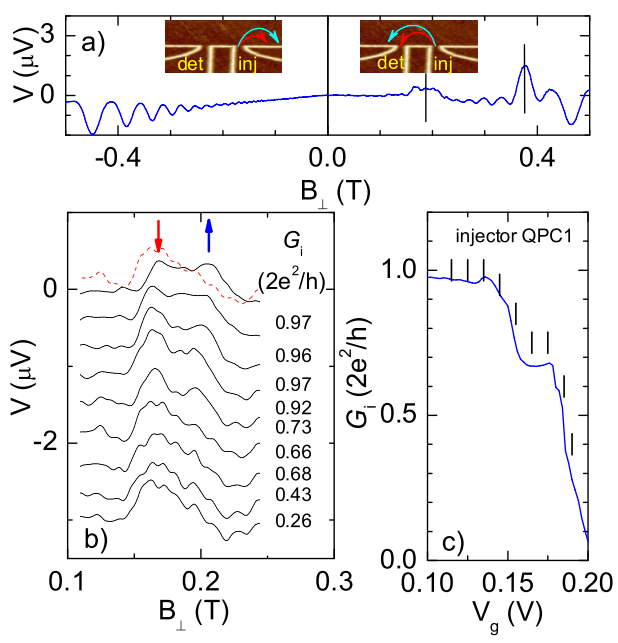

FIG. 3 (color online). Polarization detection via magnetic focusing. (a) The voltage across the detector QPC2 is measured as a function of the perpendicular magnetic field $\left(B_{\perp}\right)$. A current of $0.5 \mathrm{nA}$ is flowing through the injector QPC1. The positions of the first two magnetic focusing peaks are marked with vertical lines. The trajectories of the ballistic holes for positive and negative $B_{\perp}$ are shown schematically in the insets. (b) The first focusing peak is measured at different injector conductances with the detector tuned into the middle of the $2 e^{2} / h$ plateau. The curves are vertically offset by $-0.4 \mu \mathrm{V}$ relative to the top one. The $G=0.66 g_{0}$ curve is also plotted without an offset [dashed line (red online)]. (c) The gate voltage characteristic of QPC1. Vertical lines mark the positions where the curves in (b) are taken.

The dependence of the first focusing peak on the injector conductance is shown in Fig. 3(b). The top curve is measured with the conductance of both QPC1 and QPC2 tuned into the first quantized plateau $G=g_{0}=2 e^{2} / h$. Both peaks have approximately the same value, consistent with the expectation that at $G=g_{0}$ there are two fully transmitting spin states below the Fermi energy. We fix the detector QPC2 at $G_{d}=g_{0}$ to allow both spin states to be detected and gradually reduce the conductance of the injector QPC1 to $G_{i}<g_{0}$. As $G$ decreases, the height of the high- $B$ peak within the first focusing peak decreases, while the height of the low- $B$ peak increases. This indicates that the two subbands with opposite spins are not equally populated at $G<g_{0}$ and, thus, there is a finite polarization of holes injected from QPC1. We estimate $P_{i} \approx 40 \pm 15 \%$ for $G<0.9 g_{0}$ using either the ratio of the two peaks or suppression/enhancement of each peak. Note that the polarization due to Zeeman splitting of the spin subbands in an external magnetic field is too small to be detected in our experiments, $g^{*} \mu_{B} B_{\perp} \approx 6 \mu \mathrm{eV} \lesssim k_{B} T, e V_{a c}$. Also, we do not expect the hyper-fine interaction to play a significant role since the leading contact-Fermi term is absent for holes.

The appearance of a plateau around $0.7 g_{0}$ requires substantial energy splitting between the two spin subbands, comparable to or larger than the level broadening. In many
QPCs, though, this condition is not met and there is no extra plateau below $g_{0}$. The question remains whether there is still a finite polarization below the first quantized plateau. We investigated several QPCs with no 0.7 structure; see Fig. 4. The samples are fabricated from different wafers A (left panel) and B (right panel). The injector QPCs in both devices have well-defined first quantized plateau at $2 e^{2} / h$ but no 0.7 structure. The magnetic focusing signal is measured with the detector QPC fixed at $G_{d}=$ $g_{0}$. At $G_{i}=g_{0}$ the first focusing peak is split in two peaks of similar height, with both spin subbands being populated. As $G_{i}$ is decreased below $g_{0}$, one of the peaks becomes suppressed while the other is enhanced, similar to the device with a well-defined 0.7 structure. The polarization $P_{i}$ increases gradually from 0 to $\sim 15 \%$ as $G_{i}$ decreases from $1 g_{0}$ to $0.2 g_{0}$; the polarization is approximately 2 times lower than in the device with the 0.7 structure. We conclude that the polarization of QPCs near the onset of conduction is a rather generic property and that the appearance of the 0.7 structure is an extreme indicator of such polarization when the spin gap becomes large enough to result in a measurable feature in the gate voltage characteristic.

The two devices in Fig. 4 have different crystallographic orientations and, thus, different angles between the momentum of the injected carriers and the internal spin-orbit field $\left(I_{i} \|[\overline{2} 33]\right.$ and $I_{i} \|[0 \overline{1} 1]$ for the samples on the left and right panels, respectively) which, presumably, results in a different peak being suppressed. For the device to work as a spin detector it is sufficient that each spin state in a QPC adiabatically maps onto one of the chiral states in

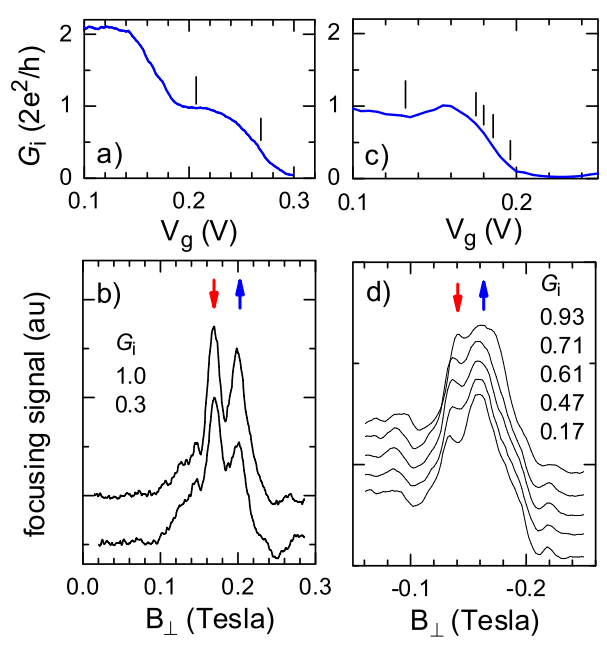

FIG. 4 (color online). Polarization in samples with no welldefined 0.7 structure. (a), (c) Conductance of the injector QPCs for two samples. (b), (d) The first focusing peak is plotted for fixed $G_{d}=2 e^{2} / h$ and $G_{i}$ as indicated in the labels (in units of $2 e^{2} / h$ ). Vertical lines in (a), (c) mark the positions where the corresponding curves in (b), (d) are taken. Curves in (b), (d) are offset for clarity. 
the adjacent 2D gas, which has been checked by the application of a strong Zeeman field as discussed in Ref. [10]. The exact mapping conditions are the subject of ongoing research.

We conducted several tests to ensure that the extracted polarization is not dominated by disorder-mediated fluctuations. The reported data were reproducible over several thermal cyclings to room temperature (six for the sample in Fig. 3). Switching the injector and detector with a simultaneous reversal of the magnetic field results in almost identical magnetic focusing data. The $P_{i}$ calculated from each peak at the same $B$ is consistent with the $P_{i}$ calculated from both peaks $\Delta B=30 \mathrm{mT}$ apart, which is comparable with the period of the mesoscopic fluctuations in similar structures. Asymmetric gating of the point contact shifts the conducting channel in space and, thus, allows us to scan through the underlying disorder potential [28]. Changing $V_{\mathrm{gc}}-V_{g 1}$ by $90 \mathrm{mV}$ shifts the channel by $\approx 7 \mathrm{~nm}$, while the correlation length for the disorder inside a 1D channel in similar but higher mobility electron samples was measured $\approx 2 \mathrm{~nm}$. In our sample this shift also translates into an extra half-flux quantum being inserted inside the focusing trajectory. Experimentally, the peak heights remain the same as we laterally shift the injector channel (although the peaks become slightly broader). Finally, the peak height is sensitive to in-plane magnetic field (see Fig. 2 of Ref. [10]), which is expected for spin subbands but not for mesoscopic fluctuations. Thus, our experiments provide a direct measurement of finite polarization in point contacts.

In conclusion, we present an experimental investigation of the 0.7 structure in $p$-type QPCs with a new twist: a direct measurement of the spin polarization. Using a newly developed spin separation technique, we determine the polarization of the holes injected from a QPC into an adjacent $2 \mathrm{D}$ gas. The technique is sensitive to static polarization, which is found to be as high as $40 \%$ in samples with a well-defined 0.7 structure. Some polarization has been measured in all point contacts below the first plateau. This result questions the Kondo interpretation as an origin of the 0.7 structure, which is incompatible with a finite static polarization. The 0.7 structure in $p$-type QPCs shows all the essential features reported for $n$-type QPCs, such as a gradual evolution into the $0.5 g_{0}$ plateau at high in-plane magnetic fields, survival at high temperatures, a gradual increase toward $1.0 g_{0}$ at low temperatures, and the zerobias anomaly, which is suppressed by either temperature increase or application of a magnetic filed. The similarities between $p$-type and $n$-type QPCs suggest that the underlying physics responsible for the appearance of the 0.7 structure should be the same.

The authors thank Yu. Lyanda-Geller and G. F. Giuliani for discussions. This work was supported by NSF Grant No. ECS-0348289.

[1] D. Weinmann, W. Häusler, and B. Kramer, Phys. Rev. Lett. 74, 984 (1995).

[2] L. P. Rokhinson, L. J. Guo, S. Y. Chou, and D.C. Tsui, Phys. Rev. B 63, 035321 (2001).

[3] A. K. Huttel et al., Europhys. Lett. 62, 712 (2003).

[4] S. Papadakis et al., Science 283, 2056 (1999).

[5] K. J. Thomas et al., Phys. Rev. Lett. 77, 135 (1996).

[6] A. Kristensen et al., Phys. Rev. B 62, 10950 (2000).

[7] S. M. Cronenwett et al., Phys. Rev. Lett. 88, 226805 (2002).

[8] P. Roche et al., Phys. Rev. Lett. 93, 116602 (2004).

[9] Y. V. Sharvin, Zh. Eksp. Teor. Fiz. 48, 984 (1965) [Sov. Phys. JETP 21, 655 (1965)]; V. S. Tsoi, Zh. Eksp. Teor. Fiz. 68, 1849 (1975) [JETP Lett. 22, 197 (1975)]; H. van Houten et al., Phys. Rev. B 39, 8556 (1989).

[10] L. P. Rokhinson et al., Phys. Rev. Lett. 93, 146601 (2004).

[11] B. J. van Wees et al., Phys. Rev. Lett. 60, 848 (1988).

[12] D. A. Wharam et al., J. Phys. C 21, L209 (1988).

[13] N. Bagraev et al., Semiconductors 36, 439 (2002).

[14] H. Chou et al., Appl. Phys. Lett. 86, 073108 (2005).

[15] R. de Picciotto, L. Pfeiffer, K. Baldwin, and K. West, Phys. Rev. Lett. 92, 036805 (2004).

[16] D. J. Reilly et al., Phys. Rev. Lett. 89, 246801 (2002).

[17] E. Lieb and D. Mattis, Phys. Rev. 125, 164 (1962).

[18] B. Spivak and F. Zhou, Phys. Rev. B 61, 16730 (2000).

[19] K. F. Berggren and I. I. Yakimenko, Phys. Rev. B 66, 085323 (2002).

[20] A. D. Klironomos, J. S. Meyer, and K. A. Matveev, condmat/0507387.

[21] Y. Meir, K. Hirose, and N. S. Wingreen, Phys. Rev. Lett. 89, 196802 (2002).

[22] G. Seelig and K. A. Matveev, Phys. Rev. Lett. 90, 176804 (2003).

[23] K. A. Matveev, Phys. Rev. Lett. 92, 106801 (2004).

[24] E. S. Snow and P. M. Campbell, Appl. Phys. Lett. 64, 1932 (1994).

[25] R. Held et al., Appl. Phys. Lett. 73, 262 (1998).

[26] L. P. Rokhinson, D. C. Tsui, L. N. Pfeiffer, and K. W. West, Superlattices Microstruct. 32, 99 (2002).

[27] R. M. Potok, J. A. Folk, C. M. Marcus, and V. Umansky, Phys. Rev. Lett. 89, 266602 (2002).

[28] T. Heinzel et al., Phys. Rev. B 61, R13353 (2000). 